\title{
Doença inflamatória intestinal: percepções dos pacientes e dos profissionais de saúde sobre a tomada de decisão compartilhada
} Inflammatory bowel disease: patients' and professionals' perceptions of shared decision-making Enfermedad inflamatoria intestinal: percepciones de los pacientes y de los profesionales de la salud sobre la toma de decisiones compartida

\author{
María Carmen Villar Bustos ${ }^{1}$ i nttps://orcid.org/0000-0002-4023-5651
}

Elena Andina-Díaz² io https://orcid.org/0000-0001-9687-1967

\section{Como citar:}

Villar Bustos MC, Andina-Díaz E. Doença inflamatória intestinal: percepções dos pacientes e dos profissionais de saúde sobre a tomada de decisão compartilhada. Acta Paul Enferm. 2021;34:eAPE000765.

DOI

http://dx.doi.org/10.37689/actaape/2021A0000765

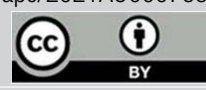

Descritores

Decisões; Doenças inflamatórias intestinais; Continuidade da assistência ao paciente

Keywords

Shared decision-making; Inflammatory bowel disease; Continuity of patient care

Descriptores

Decisiones; Enfermedades inflamatorias de intestino; Continuidad de la atención al paciente

Submetido

7 de Abril de 2020

Aceito

1 de Março de 2021

\section{Autor correspondente}

Carmen Villar Bustos E-mail: mcvillarb@gmail.com

\section{Resumo}

Objetivo: Descrever as percepções de implementação da lei 41/2002 sobre a autonomia do paciente com doença inflamatória intestinal e profissionais em relação à tomada de decisão compartilhada.

Métodos: Estudo qualitativo e descritivo, utilizando abordagem fenomenológica. Foram realizadas entrevistas semiestruturadas em dez pacientes pertencentes à Associação de Pacientes com Doença de Crohn e Colite Ulcerativa de Zamora (Espanha), e grupo focal com sete gastroenterologistas e enfermeiros da clínica de ostomia e unidade de gastroenterologia hospitalar do Hospital Virgen de la Concha de Zamora. Foi realizada análise do conteúdo temático dos dados.

Resultados: Surgiram duas categorias principais e sete subcategorias: Informação (com conhecimento dos profissionais e dos pacientes, confiança no profissional, tempo e atitude em relação à informação) e Tomada de decisão compartilhada (com atitude em relação à informação, enfrentamento/resignação e apoio às decisões). A tomada de decisão compartilhada é um processo complexo, onde não somente a informação é 0 principal benefício para os pacientes, mas outras questões como apoio, tempo de doença ou emergência são importantes para a decisão do paciente.

Conclusão: Descrever as percepções dos pacientes e dos profissionais sobre a tomada de decisões compartilhadas, que as viam como um processo complexo. Melhorar as informações fornecidas aos pacientes sobre sua doença e seus direitos pode influenciar a participação na tomada de decisão compartilhada e modificar atitudes. Os pacientes não adotaram permanentemente um papel ativo ou passivo em relação à tomada de decisão compartilhada, mas, por múltiplos fatores, oscilaram entre os papéis. Consequentemente, é importante que os profissionais de saúde se envolvam no processo de compreensão dos pacientes e de suas necessidades para facilitar a tomada de decisão compartilhada. Além disso, é necessário maior investimento por parte das autoridades para garantir continuidade dos cuidados e desenvolver unidades multidisciplinares para doenças inflamatórias intestinais como forma de melhorar a tomada de decisão compartilhada.

\section{Abstract}

Objective: To describe perceptions of implementing law 41/2002 on patient autonomy in inflammatory bowel disease patients and professionals in relation to shared decision-making.

Methods: Qualitative descriptive study using a phenomenological approach. We conduced semi-structured interviews to 10 patients belonging to the Association of Patients with Crohn's Disease and Ulcerative Colitis in Zamora (Spain) as well as focus group to 7 gastroenterologists and nurses from the ostomy clinic and inpatient gastroenterology unit at the Virgen de la Concha Hospital in Zamora. Data thematic content analysis was performed. 
Results: Two main categories and seven sub-categories emerged: Information (with professionals' and patients' knowledge, trust in the professional, time and attitude to information) and Shared decision-making (with attitude to information, coping-resignation and support for decisions). Shared decision-making is a complex process where not only the information is the main value for the patients, but other issues like support, time of illness or emergency are important for patient decision.

Conclusion: To describe patients' and professionals' perceptions of shared decision-making, who saw it as a complex process. To improve the information provided to patients about their illness and their rights can influence participation in shared decision-making and change attitudes. Patients did not permanently adopt an active or passive role in relation to shared decision-making, but instead oscillated between roles depending on multiple factors. Consequently, it is important for health professionals to engage in the process of understanding patients and their needs to facilitate shared decision-making. In addition, greater investment by the authorities is needed to ensure continuity of care and create multidisciplinary inflammatory bowel disease units as measures to improve shared decision-making.

\section{Resumen}

Objetivo: Describir las percepciones de la implementación de la ley 41/2002 sobre la autonomía del paciente con enfermedad inflamatoria intestinal y profesionales con relación a la toma de decisiones compartida.

Métodos: Estudio cualitativo y descriptivo, que utiliza el enfoque fenomenológico. Se realizaron entrevistas semiestructuradas a diez pacientes pertenecientes a la Asociación de Pacientes con Enfermedad de Crohn y Colitis Ulcerosa de Zamora (España); y un grupo focal de siete gastroenterólogos y enfermeros de la clínica de ostomía y de la unidad de gastroenterología hospitalaria del Hospital Virgen de la Concha de Zamora. Se realizó un análisis del contenido temático de los datos.

Resultados: Surgieron dos categorías principales y siete subcategorías: Información (con conocimiento de los profesionales y de los pacientes, confianza en el profesional, tiempo y actitud con relación a la información) y Toma de decisiones compartida (con actitud respecto a la información, enfrentamiento/ resignación y apoyo a las decisiones). La toma de decisiones compartida es un proceso complejo en el que no solamente la información es el principal beneficio para los pacientes, sino que también hay otras cuestiones importantes para la decisión del paciente, como el apoyo, el tiempo de enfermedad o la emergencia.

Conclusión: Describir las percepciones de los pacientes y de los profesionales sobre la toma de decisiones compartida, que era vista como un proceso complejo. Mejorar la información ofrecida a los pacientes sobre su enfermedad y sus derechos puede influir en la participación de la toma de decisiones compartida y modificar actitudes. Los pacientes no adoptaron un papel permanentemente activo o pasivo respecto a la toma de decisiones compartida, sino que oscilaban entre los dos papeles debido a múltiples factores. Por lo tanto, es importante que los profesionales de la salud se involucren en el proceso de comprensión de los pacientes y de sus necesidades para facilitar la toma de decisiones compartida. Además, son necesarias mayores inversiones por parte de las autoridades para garantizar una continuidad de los cuidados y desarrollar unidades multidisciplinarias para enfermedades inflamatorias intestinales como forma de mejorar la toma de decisiones compartida.

\section{Introdução}

As últimas décadas testemunharam o surgimento de uma tendência à participação do paciente, com base na ideia de que o conhecimento especializado no paciente e a experiência no gerenciamento de sua doença devem ser utilizados na tomada de decisão em relaçáo ao seu tratamento e poderia incentivar outros a tomar decisóes cruciais durante o processo de cuidado. Esse fato tem o potencial de aumentar a satisfação pessoal, melhorar a qualidade de vida e o gerenciamento da doença. ${ }^{(1,2)}$

Entretanto, alguns estudos relatam que na prática, os pacientes podem achar difícil assegurar sua autonomia, pois ter um controle maior da tomada de decisóes que afetam sua saúde pode ser vista negativamente pelos próprios pacientes ou por profissionais. ${ }^{(2)}$ Os pacientes querem ser bem informados, mas o direito de participar de seu tratamento tomando decisões autônomas não é visto como uma necessidade e eles geralmente preferem deixar a tomada de decisão a cargo de seu médico, conforme evidenciado por González Mestre. ${ }^{(2)}$
A Tomada de Decisão Compartilhada (TDC) é um processo colaborativo, no qual pacientes e profissionais de saúde trocam informaçóes (pessoais e médicas), discutem e chegam a uma decisão consensual. Profissionais e pacientes trabalham juntos para escolher entre as opçóes terapêuticas ou preventivas disponíveis, com base nas evidências científicas, selecionando a que melhor se adapta às preferências e valores do paciente, o que inclui também a opção de não fazer nada. ${ }^{(3,4)}$

As principais barreiras à implementação do TDC incluem falta de tempo e recursos no sistema de saúde, resistência e ceticismo. ${ }^{(5)} \mathrm{O}$ uso das habilidades de comunicação como escuta ativa e empatia, incentiva a participação do paciente neste processo ${ }^{(5,6)}$ e é essencial para sua implementaçáo. ${ }^{(7,8)}$ A motivação do paciente e a percepção do impacto positivo do processo e seus resultados também facilitam o TDC. ${ }^{(9)}$

A participação do paciente na TDC está associada a uma maior satisfação com o tratamento e níveis mais baixos de ansiedade em relaçáo aos procedi- 
mentos médicos, o que melhora a aderência ao tratamento. ${ }^{(9)}$ Isto pode variar de acordo com nível de educaçáo, consciência de saúde e idade dos pacientes, e é significativamente maior nos jovens. ${ }^{(6)}$ Outros estudos têm demonstrado que as decisóes mais importantes suscitam uma participação mais ativa. ${ }^{(7,10)}$

A participação dos pacientes em assuntos da sua doença e na TDC varia muito de acordo com o país. Por exemplo, alguns estudos europeus relataram diferenças em fatores atribuíveis tanto a profissionais quanto a pacientes. ${ }^{(11)} \mathrm{Na}$ Espanha, onde o presente estudo foi realizado, a lei 41/2002 sobre autonomia do paciente e direitos e obrigaçóes relacionadas à informação e documentação médica na área da saú$\mathrm{de}^{(12)}$ estabelece o direito legal à autonomia do paciente como princípio básico.

A Doença Inflamatória Intestinal (DII) é uma doença crônica de causa desconhecida e prognóstico incerto. Engloba várias patologias autoimunes que causam inflamação crônica do intestino e apresentam crises de atividade inflamatória (fase ativa) com períodos de remissão (fase dormente). As formas mais severas e também mais prevalentes são a Doença de Crohn (DC) e a Colite Ulcerativa (CU), com idade média ao diagnóstico de 30 anos. ${ }^{(13)} \mathrm{A}$ incidência de DII está aumentando na Europa e é atualmente estimada em 24,3/100.000 habitantes/ano para CU e 12,7/100.000 habitantes/ano para DC. ${ }^{(14)} \mathrm{Na}$ Espanha, as estimativas são de 5,6-9,4/100.000 habitantes/ano para CU e 8,9-10,8/100.000 habitantes/ ano para DC. ${ }^{(15)}$ Em 2015, o custo direto anual por paciente com CU na Espanha foi de $€ 1754,10$ euros (custo indireto: $€ 399,32$ euros), ${ }^{(16)}$ e um relatório em 2016 estimou um custo total anual de 1,8\% dos gastos com saúde pública. ${ }^{(13)}$

Neste estudo, examinamos o conhecimento e a aplicação da lei 41/2002 no cuidado de saúde de pacientes com DII, uma doença crônica altamente complexa que se apresenta em uma idade precoce, e na qual os pacientes participam de múltiplas situaçóes relacionadas ao TDC devido a sua longa experiência com a doença. Poucos estudos analisando as percepçóes dos pacientes e profissionais em relação ao TDC foram encontrados na literatura; ${ }^{(6-8)}$ assim, seria útil identificar os pontos de vista de ambos os grupos a fim de esclarecer as razóes para a participação dos pacientes.
Considerando o exposto, o objetivo deste estudo foi descrever as percepçóes de implementação da lei 41/2002 sobre a autonomia dos pacientes em pacientes e profissionais do DII em relação à TDC.

\section{Métodos}

Estudo qualitativo e descritivo, utilizando uma abordagem fenomenológica. O objetivo da fenomenologia é descrever e esclarecer o significado de fenômenos particulares, ou a aparência das coisas, como experiência vivida. É a experiência vivida que dá sentido à percepção de cada indivíduo de um fenômeno particular. $^{(17)}$ Assim, uma abordagem fenomenológica permitiu analisar as percepçóes de pacientes e profissionais em relação aos fenômenos TDC.

$O$ estudo foi realizado na cidade de Zamora (Espanha). A população do estudo consistiu de pacientes pertencentes à Associação de Pacientes com Doença de Crohn e Colite Ulcerativa (iniciais espanholas: ACCU), em Zamora, assim como gastroenterologistas e enfermeiros da clínica de ostomia e da unidade de gastrenterologia hospitalar de Virgen de la Concha, em Zamora. Para acessar o grupo de pacientes, contatamos o presidente da ACCU Zamora (presencialmente); enquanto que para o grupo de profissionais foi contatado o chefe da unidade e supervisor de enfermagem da unidade de gastrenterologia do Hospital Virgen de la Concha (presencialmente). Estes facilitaram o primeiro acesso aos participantes e auxiliaram na condução da amostragem teórica e, posteriormente, a amostragem de conveniência (método bola de neve). ${ }^{(18)}$

O grupo de pacientes nos foi apresentado pelo presidente da ACCU, o que facilitou para o estabelecimento do relacionamento. No caso do grupo de profissionais, o fato do pesquisador ter sido enfermeira no hospital - embora em um serviço diferente - auxiliou no contato com os profissionais (empatia). Selecionamos indivíduos que preenchiam os critérios de inclusão (ser maior de 18 anos e não ter déficits cognitivos/doença mental [ambos os grupos]; ter ou estar relacionado a alguém com DII [grupo de pacientes]; e ter conhecimento e experiência do processo DII [grupo profissional]), e os 
convidamos a participar do estudo. Nenhum convidado se recusou a participar do estudo [ambos os grupos]. Ao selecionar os participantes, procuramos obter um número comparável de pacientes com DC e CU, assim como profissionais de diferentes categorias, alcançando uma distribuição equitativa por sexo em pacientes e profissionais.

Foram utilizadas duas técnicas para a coleta de dados. Para o grupo de pacientes, o investigador principal realizou entrevistas semiestruturadas (duração entre 27 e 69 minutos), conduzidas nas instalaçôes da ACCU Zamora, em uma sala tranquila, longe de ruídos (para facilitar contato agradável), dentro da associação (fevereiro-maio de 2018). As entrevistas foram realizadas, até que a saturação dos dados fosse atingida (dez entrevistas). Para o grupo profissional, foi realizado um grupo focal (duração de 50 minutos) na sala de reunióes da unidade de gastrenterologia do hospital (sala calma, longe do ruído), moderado pelo investigador principal (maio de 2018). Dez pacientes e sete profissionais do grupo focal foram considerados suficientes para alcance da saturação de dados. As entrevistas e o grupo focal foram gravadas em áudio e notas adicionais foram tomadas. $\mathrm{O}$ mesmo guia foi usado para entrevistas e para o grupo focal, e esta continha uma lista de perguntas para esta finalidade, de acordo com o estudo de López Cortés. ${ }^{(19)}$

Entrevistas e gravaçóes dos grupos focal foram transcritas literalmente e a análise de dados foi realizada usando os programas de software Excel $^{\circledR}$ e Weft QDA1.0.1 ${ }^{\circledR}$. Após uma leitura geral e várias leituras em profundidade, ambos os pesquisadores analisaram o conteúdo. O conteúdo foi analisado de acordo com a proposta de Giorgi. ${ }^{(20)}$ Primeiramente, foi realizada uma leitura rigorosa dos dados e a seguir, uma nova leitura a fim de extrair todas as unidades de significado. Em terceiro lugar, as unidades de significado foram agrupadas em categorias/subcategorias principais, de acordo com suas características compartilhadas. Assim, duas principais categorias emergiram dos dados (informação e TDC), sete subcategorias, e suas unidades de significado correspondentes. A análise foi obtida por consenso entre os dois membros da equipe de pesquisa.

Para garantir a validade, transcrições de áudio foram enviadas por e-mail para o grupo de pacien- tes. Eles confirmaram seus comentários e os principais temas/categorias que os pesquisadores identificaram na análise (categorias). No grupo profissional, foi realizada uma reuniáo presencial, com o mesmo objetivo. Da mesma forma, um pesquisador externo (com experiência no assunto) validou os resultados. As informaçóes contraditórias detectadas nos pronunciamento dos participantes foram esclarecidas durante estas reuniáo.

A consistência no discurso foi obtida pela triangulação de dados e métodos. Uma atitude de autocrítica foi mantida durante todo o processo. Para evitar um viés na formulação das perguntas da pesquisa (já que ambos os pesquisadores eram enfermeiros), estas foram elaboradas de acordo com estudos anteriores. ${ }^{(19)}$

Para evitar influência na coleta de dados, recrutamento de amostras e escolha do local, os pesquisadores só foram informados superficialmente sobre o tópico (como profissionais de saúde) e estes não eram sua área de trabalho/tema usual de pesquisa. Ambos os pesquisadores iniciaram a análise após a primeira entrevista, a fim de avaliar se estava de acordo com os objetivos e estarem preparados caso alguma mudança no desenho da pesquisa fosse necessária (não foi necessária).

Este estudo respeitou os requisitos éticos da Declaração de Helsinque e da lei orgânica 3/2018, em relação à proteçáo de dados pessoais e direitos digitais e foi aprovado pelos Comitês de Ética (ETHICS-ULE-001-2018 e Hospital (Zamora)).

Pacientes e profissionais receberam informaçóes detalhadas por escrito sobre o estudo e assinaram um termo de consentimento livre e esclarecido. Os pacientes foram anonimizados, recebendo número (001, etc.) e os profissionais com a letra P e um número (P1, etc.).

\section{Resultados}

A composição da amostra final é apresentada nas tabelas 1 e 2 .

Foram detectadas duas categorias principais nas quais os princípios da lei são baseados: 1) informaçôes necessárias para tomar uma decisão e 2) 
Tabela 1. Composição da amostra final (pacientes)

\begin{tabular}{|c|c|c|c|c|c|}
\hline & Sexo & Idade & $\begin{array}{c}\text { Nivel de } \\
\text { escolaridade }\end{array}$ & $\begin{array}{c}\text { Idade ao } \\
\text { diagnóstico }\end{array}$ & Tipo de doença \\
\hline 001 & M & 66 & Básico & 35 & Colite ulcerativa \\
\hline 002 & $\mathrm{~F}$ & 51 & Básico & 17 & Doença de Crohn \\
\hline 003 & $\mathrm{~F}$ & 38 & Universitário & 30 & Colite ulcerativa \\
\hline 004 & M & 23 & Universitário & 16 & Colite ulcerativa \\
\hline 005 & M & 59 & Universitário & 23 & Doença de Crohn \\
\hline 006 & $\mathrm{~F}$ & 60 & Básico & 48 & Colite ulcerativa \\
\hline 007 & $\mathrm{~F}$ & 58 & Universitário & 38 & Doença de Crohn \\
\hline 008 & $\mathrm{~F}$ & 28 & Universitário & 28 & Doença de Crohn \\
\hline 009 & M & 49 & Universitário & 36 & Colite ulcerativa \\
\hline 010 & M & 57 & Básico & 39 & Colite ulcerativa \\
\hline
\end{tabular}

Tabela 2. Composição da amostra final (profissionais)

\begin{tabular}{|c|c|c|c|}
\hline & Sexo & Idade & Profissional \\
\hline P1 & $\mathrm{F}$ & 52 & Médico gastroenterologista \\
\hline P2 & M & 44 & Médico gastroenterologista \\
\hline P3 & M & 61 & Enfermeiro \\
\hline P4 & M & 61 & Enfermeiro \\
\hline P5 & M & Não informado & Médico gastroenterologista \\
\hline P6 & M & 42 & Médico gastroenterologista \\
\hline P7 & M & 58 & Enfermeiro \\
\hline
\end{tabular}

Tomada de Decisão Compartilhada, da qual surgiram sete subcategorias, descritas abaixo.

\section{1) Informações necessárias para a tomada de uma decisão:}

A maioria dos pacientes relatou ter pouco ou nenhum conhecimento da lei $41 / 2002$, enquanto os profissionais só sabiam sobre ela em termos gerais, mas ambos os grupos disseram conhecer os direitos dos pacientes.

Não... e esta lei... por que esta lei não é relatada... aos... pacientes? (002)

Bem, eu acho que a lei do paciente que teria que... a quem teria que ser dirigida para ser estudada e colocada em prática são os próprios profissionais (006)

Os pacientes relataram ter recebido informaçóes sobre sua doença, mas, declararam que gostariam que fossem mais fáceis de entender. Também sugeriram que os profissionais deveriam escolher o melhor momento para transmitir informaçóes e considerar as características do paciente (nem todos estão igualmente receptivos à informação ou precisam saber a mesma coisa). Além disso, enfatizaram a importância de receber mais informaçóes quan- do mudou o tratamento, ou quando os sintomas pioraram.

Eles preferiram receber informações diretamente do médico em vez de outros canais. Eles consideraram a Internet uma fonte de informação não confiável e preferiram o material fornecido pela associação.

Cada paciente e cada corpo é diferente... esta doença é muito particular e afeta cada pessoa de uma forma diferente... Agora... se você disser que é meu médico, ou uma pessoa que colabora comigo, bem, veja... Eu gostaria de ter isto para mim (002)

\section{1.a Conhecimento dos pacientes e dos profissionais:}

Estas informaçóes percebidas como melhores ou piores, dependendo de conhecimento pessoal. Para os profissionais, dependendo do conhecimento da doença:

Cada paciente precisa de informaçóes particulares em um determinado momento; o DII é uma doença altamente complexa e estes pacientes são especiais (P2)

Para os pacientes, dependendo da autopercepção do conhecimento de acordo com o nível educacional e conhecimento dos direitos do paciente e de sua própria doença:

Então, tem também o nivel cultural, ajuda muito se os pacientes souberem sobre sua doença, e se tiverem uma boa compreensão do tratamento, eles acham isso muito mais fácil (PG)

Eu acho que a lei sobre os pacientes deve ser.. deve ser dirigida e estudada e posta em prática pelos próprios profissionais (005)

Eles acham que sabem mais do que realmente sabem, porque quando você começa a explicar a eles... (P3)

E por último, dependendo do conhecimento de seus pares (associaçóes no caso de pacientes, outros profissionais no caso de profissionais): 
Na verdade, foi graças à associação que eu descobri mais sobre a doença e aprendi sobre todos os sintomas (004)

Primeiro, em geral, eles não se percebem como capazes, são pacientes muito complexos e difíceis. Eles estão assustados (PG)

\section{1.b Confiança no profissional:}

O relacionamento estabelecido entre profissional e paciente influenciou as percepçóes das informaçóes recebidas, sendo que quanto mais confiável era o profissional, mais positivas eram as percepçóes das informaçóes recebidas:

Depende também dos profissionais... quando você entra, eles estão escrevendo, não olham para você... e ainda nem sabem seu nome... (005)

Parecia errado que eu estivesse pedindo meus próprios registros... Quero dizer... o que eu deveria ter feito era informa-los por não tê-los entregue a mim, porque são meus... (004) Confio no médico, é sempre o que o médico diz (006)

Sempre confiei no médico porque, dentre outras coisas, náo tive opção... (002)

A informação era melhor recebida se o profissional mostrasse empatia:

Para os pacientes, um tapinha nas costas é muito mais eficaz... / palavras tranquilizadoras faz muito mais do que a medicina (001)

\section{1.c Tempo:}

Falta de tempo foi considerada uma barreira importante para o fornecimento de informaçóes, considerando dois momentos diferentes: o momento da doença (por exemplo, uma recaída):

Quando você chega aqui as filas são enormes, o médico está estressado e quase não tem tempo para... acho que a única coisa é que deveríamos ter mais tempo, não só nós, mas eles também, os médicos, que não podem nos dar tanto tempo quanto gostariam (006)
Sou o tipo de pessoa que precisa de tempo para pensar nas coisas... le depois, absorver... Eu pensaria em outras perguntas... mas não posso perguntar a ninguém porque já estou no caminho de volta para casa (002)

E o tempo gasto durante uma consulta:

Vejamos, a principio eu me lembro que quando me falaram em ter uma ostomia e uma operação urgente, bem... Eu bati os pés, não queria saber disso (004)

Quando são admitidos no hospital é por causa de uma recaída e às vezes não há opçóes (P6)

Uma unidade para DII seria ótimo, porque o que esses pacientes querem é continuidade e obviamente, você nem sempre estamos lá... (P1)

É colite, não é mais nada... e, claro, você está aliviado, mas só... mais tarde, quando você descobre mais sobre isso, é outra história!... Não é a palavra mais assustadora, como câncer, por exemplo, mas não há cura... (010)

\section{1.d Atitude à informaçáo:}

Depende da atitude dos profissionais em fornecer informaçóes, por exemplo, habilidades para comunicação, desejo de informar:

possivel facilitar a compreensão? Bem... eu não diria não... talvez... (P1)

$E$, sobre as atitudes dos pacientes para recebe-las (querendo ser informado):

Não é preciso muita informação no início... quando se está... assimilando... (003)

$E$ às vezes eles the dizem que é melhor não ler porque se o fizer, eu não vou assinar. Porque com o que eles me dizem aqui... (P3)

Ambos os aspectos foram considerados fundamentais em todo o processo. 


\section{2) Tomada de decisão compartilhada:}

Esta estava diretamente relacionada às percepçóes da qualidade das informaçóes recebidas. Para os pacientes, a confiança de que o profissional desempenhou um papel importante ao optar pela TDC, considerando que o profissional deve ser aquele "quem sabe", aquele que toma a decisão final ou a responsabilidade junto com os pacientes.

Eu sou o paciente e ela... a médica, a que entende mais sobre o assunto, ela é a que tem um pouco mais a dizer (008)

\section{2.a Atitude em relaçáo à TDC:}

Os pacientes relataram ver a TDC como uma responsabilidade compartilhada.

Às vezes existem opçóes... /você pode pensar em continuar com o que eles estáo tomando e esperar um pouco mais, ou quando introduzir um novo tratamento, então eles serão capazes de tomar uma decisão (P2)

Todavia, eles se sentiriam melhor preparados para tomar uma decisão importante, "mesmo contra o conselho médico", se tivessem mais informaçóes sobre o assunto:

A tomada de decisão deve ser apoiada por muitas e muitas informaçôes... então você pode decidir (004) É errado dizer isso... mas já houve momentos em que eu não estava tomando nada, e fui eu quem tomou essa decisão sem discuti-la... (003)

Enquanto isso, os profissionais relataram que a TDC deveria ser uma decisão consensual com o paciente:

Às vezes é possivel decidir entre várias opçôes, mas outras vezes não é (P6)

Apesar disso, eles mantiveram uma atitude um tanto paternalista:

Seria uma situação em que você explica tudo ao paciente e depois o paciente decide... mas no final é você quem o direciona (P1)

\section{2.b Enfrentamento-resignação:}

Um fator considerado chave na TDC, tanto por pacientes quanto profissionais, foi a capacidade de enfrentamento/resignação, como características de um paciente ativo/passivo. Isto é determinado pelo estágio, cronicidade ou complexidade da doença:

Porque, se você tem muitas recaídas, isso o esgota (005)

O tempo passa e você percebe que não está melhorando... você não gosta disso, mas bem... isso é o que há e você tem que viver com isso, obviamente... (010)

Eu tive isso por quatro anos, entre uma coisa e outra... Vou a um médico que me diz que esta é a doença de Crohn, vou a outro que não tem tanta certeza...le enquanto isso eu tenho todos esses sintomas... (008)

Às vezes, também é determinado pela incerteza, sobre se algo funciona, sobre por onde começar, sobre o prognóstico ou sobre novos tratamentos:

Sim, mas é diferente com outras doenças crônicas... por causa da complexidade desta... Outras vezes não há opçóes, não há alternativa, você tem que tratá-la, não há outra opção... não há alternativa (P1)

E não funciona, você pode estar tomando isto continuamente, está funcionando bem, e por alguma razão você para de tomarl... e depois de dois anos, talvez, ela volta... não tem funcionado... (005)

Até o médico já me disse muitas vezes... "Não me sinto capaz de controlar seu caso"... então é um pouco preocupante.. (008)

Alguns membros estão em remissão há um ano, talvez e, de repente, têm uma terrivel recaida... o que eles podem fazer? (004)

Eu tentei os últimos tratamentos que sairam mas não funcionaram... (010) 


\section{2.c Apoio às decisóes:}

Os pacientes relataram que suas redes de apoio (parceiro, família, autoridades, a equipe multidisciplinar e a associação de pacientes) também contribuíram para a TDC.

Quanto à família, parceiro ou amigos, e associação de pacientes:

Seu parceiro também tem uma enorme influência... quando seu parceiro te apoia, quando você se sente acompanhado por seu parceiro ou quando as coisas não estão indo bem com seu parceiro... (009)

muito apoio de amigos, colegas, outros da associação (008)

Às vezes nos ajudávamos uns aos outros, "venha dormir em minha casa, venha tomar um banho, descansar"... este foi um aspecto muito importante do que tivemos na associação (005)

Tanto os pacientes quanto os profissionais concordaram que era necessário mais apoio das autoridades para melhorar as consultas médicas, aumentando o tempo previsto e garantindo a continuidade do atendimento.

Mais recursos humanos, mais protocolos e mais aderência aos protocolos. Horários de consultas menos apertados (PG)

Eles informaram que uma boa solução seria ter uma unidade especializada em DII, com equipe multidisciplinar para atender à demanda de continuidade e especialização. Ambos os grupos relataram falta de interesse por parte das autoridades:

Deveria ser uma unidade multidisciplinar, onde todos os processos estejam totalmente integrados, um cirurgiáo com um radiologista porque senão, náo vale a pena (P2)

É verdade que poderiamos ter muito, mas não há uma unidade especializada em Zamora, então... (003)

\section{Discussão}

Os dados obtidos nos permitiram apresentar as percepçóes dos pacientes e dos profissionais sobre a TDC, que a consideraram um processo complexo. Esta é uma descoberta interessante, dado o número limitado de estudos identificados que exploraram esta questão em profundidade. ${ }^{(6-8)}$

Nas últimas décadas, a participação dos pacientes tem sido incentivada em vários contextos, a TDC pode levar a um aumento da satisfação dos pacientes, ajudando a melhorar a qualidade de vida e o gerenciamento das doenças. ${ }^{(1,2)}$

Os dados obtidos em nosso estudo revelam que, embora a lei 41/2002 esteja em vigor na Espanha desde 2002, poucos de nossos participantes sabiam sobre ela. A lei 41/2002 estabelece que as informaçôes fornecidas aos pacientes durante sua doença devem ser fáceis de entender para ajudá-los a tomar decisões, ${ }^{(12)}$ no entanto, nossos resultados indicam que isto ainda náo foi alcançado de forma satisfatória.

Os fatores mais importantes que influenciaram a avaliação dos participantes sobre as informaçóes recebidas foram, a confiança no profissional, o momento da doença e o conhecimento sobre o processo por parte do paciente e do profissional. Outros estudos identificaram a falta de tempo como a principal barreira relatada, tanto por profissionais quanto por pacientes. ${ }^{(7,10)}$

Nossos resultados coincidem com os encontrados na literatura ${ }^{(21)}$ e indicam que os pacientes queriam informaçóes mais completas sobre sua doença e, relataram que os profissionais precisavam de habilidades de comunicação e também selecionar o momento certo para fornecer informaçóes. Eles também preferiram receber informaçôes diretamente de um profissional e não de outras fontes.

Estudos similares $^{(7)}$ relataram a preferência dos pacientes por receber informaçóes diretamente de um especialista ou de seu médico de família, indicando que as informaçóes obtidas na Internet ou em folhetos são úteis, mas podem gerar mais ansiedade em alguns pacientes.

Nossos resultados mostram que a qualidade da informação e a confiança entre profissional e paciente influenciaram diretamente a participação na 
TDC, corroborando outros estudos realizados em diferentes contextos na Espanha. ${ }^{(22,23)}$

Nossos participantes também identificaram outros fatores que poderiam influenciar a capacidade da TDC, incluindo o estágio da doença, o conhecimento adquirido, fatores emocionais, redes de apoio e o grau de enfrentamento/resignação. A este respeito, Martin-Fernández $z^{(7)}$ explicou que o impacto da decisão, responsabilidade compartilhada com o apoio profissional e familiar são fatores que facilitam este processo.

Estudos em outros países ${ }^{(24)}$ também concluíram que somente a informação é insuficiente, e que outros fatores muito importantes devem ser levados em conta para que a TDC seja eficaz. Nem mesmo o apoio à TDC em doenças crônicas tem se mostrado eficaz por si só. ${ }^{(25)}$

Uma descoberta importante de nosso estudo foi que os pacientes apresentavam diferentes atitudes em relação à TDC. Devido a fatores como os descritos acima, os pacientes oscilaram entre os papéis ativos e passivos ao longo do curso de sua doença. Em outras palavras, não existe algo como um paciente permanentemente ativo ou passivo; ao contrário, este papel muda ao longo do curso da doença.

Finalmente, nossos resultados são consistentes com aqueles relatados em uma recente revisão sistemática de estudos sobre diferentes sistemas e culturas de saúde, ${ }^{(23)}$ que constataram que intervenções como ferramentas de ajuda não parecem aumentar o TDC em comparação com a atenção direta dos profissionais de saúde.

As limitaçóes de nosso estudo incluem o fato deste ter sido baseado nas percepçóes de pacientes com uma doença específica (pertencentes a uma associação) e de profissionais que trabalham exclusivamente no atendimento hospitalar. Pesquisas futuras devem ampliar os participantes do estudo e incluir pacientes com outras doenças crônicas e profissionais de cuidados primários, tanto na Espanha como em outros países.

\section{Conclusão}

O objetivo do estudo, ou seja, descrever as percepçóes da aplicação da lei 41/2002 quanto à autonomia dos pacientes do DII e dos profissionais em relaçáo ao TDC, foi alcançado. A TDC foi revelada como um processo complexo, facilitado pela confiança e empatia com o profissional. Além disso, parece que os pacientes necessitam de mais informaçóes sobre as implicaçôes da lei 41/2002. Nossos resultados são semelhantes aos de estudos publicados em outros países e demonstraram que, melhorar a informaçáo fornecida aos pacientes sobre sua doença e seus direitos pode influenciar a sua participação na TDC e modificar atitudes. De acordo com os pacientes, tal informação é melhor transmitida verbalmente, complementada com outros tipos de suporte. Neste sentido, os profissionais precisam melhorar suas habilidades de comunicaçáo. Constatamos que os pacientes não adotaram permanentemente um papel ativo ou passivo em relaçáo à TDC, mas sim oscilaram entre papéis dependendo de múltiplos fatores. Consequentemente, é importante que os profissionais de saúde se envolvam no processo de compreensão dos pacientes e de suas necessidades a fim de facilitar a TDC. Além disso, é necessário um maior investimento das autoridades para garantir a continuidade dos cuidados e criar unidades multidisciplinares de DII como medidas para melhorar a TDC.

\section{Colaborações}

Bustos MCV and Andina-Díaz E participaram do conceito e desenho do presente estudo; análise e interpretação dos dados; rascunho ou revisão do manuscrito e aprovaram o manuscrito conforme submetido. Todos os autores são responsáveis pela pesquisa relatada.

\section{Referências}

1. World Health Organization (WHO). Regional Office for Europe. A European policy framework and strategy for the 21st century. Health 2020: policy framework and strategy. Geneva: WHO; 2013.

2. González Mestre A. La autonomía del paciente con enfermedades crónicas: de paciente pasivo a paciente activo. Enferm Clin. 2014;24(1):67-73.

3. Participación del paciente en la toma de decisiones. Boletin Infac. 2014;22(3):1-6 [citado en 2018 Ago 1]. País Basco: Servicio Central de Publicaciones del Gobierno Vasco; 2014. Disponible: https://www. euskadi.eus/contenidos/informacion/cevime_infac_2014a/es_def/ adjuntos/INFAC_Vol_22_n_3_Decisiones_compartidas.pdf 
4. Pons Ràfols JM, Moharra Francés M. Decisiones compartidas [editorial]. Med Clin (Barc). 2016;146(5):205-6.

5. Ruiz-Azarola A, Perestelo-Pérez L. Participación ciudadana en salud: formación y toma de decisiones compartida. Informe SESPAS 2012. Gac Sanit. 2012;26(S):158-61.

6. Ruiz Moral R, Peralta Munguia L, Pérula de Torres LÁ, Olloqui Mundet J, Carrión de la Fuente T, Sobrino López A, et al. Opiniones y percepciones de los pacientes sobre su participación en la toma de decisiones en las consultas de medicina de familia. Aten Primaria. 201;4):5-10.

7. Martín-Fernández R, Abt-Sacks A, Perestelo-Perez L, Serrano-Aguilar P. Actitudes de las mujeres diagnosticadas de cáncer de mama frente a la toma de decisiones. Rev Esp Salud Publica. 2013;87(1):59-72.

8. Truglio-Londrigan M, Slyer JT, Singleton JK, Worral PS. A qualitative systematic review of internal and external influences on shared decision-making in all health care settings. JBI Database Syst Rev Implement Reports. 2014;12(5):121-94.

9. Bravo P, Contreras A, Perestelo-Pérez L, Pérez-Ramos J, Málaga G. En busca de una salud más participativa: compartiendo decisiones de salud. Rev Peru Med Exp Salud Publica. 2013;30(4):691-7.

10. Ortega-Moreno M, Padilla-Garrido N, Huelva-López L, AguadoCorrea F, Bayo-Calero J, Bayo-Lozano E. Barreras y facilitadores para la implementación de la toma de decisiones compartidas en oncología: percepciones de los pacientes. Rev Calid Asist. 2017;32(3):141-5.

11. European Commission. Eurobarometer Qualitative Study. Patient involvement. Aggregate Report [Internet]. Belgium: European Commission; 2012 [cited 2021 Feb 14]. Available from: https:// ec.europa.eu/eip/ageing/file/336/download_en\%3Ftoken=6-zWlwfx

12. España. Gobierno de España. Ley 41/2002 de 14 de noviembre, Básica Reguladora de la Autonomía del Paciente y de Derechos y Obligaciones en Materia de Información y Documentación clínica. Boletín Oficial del Estado. 2002;274 de 15/11/2002. España: Jefatura del Estado; 2002 [citado en 2021 Feb 14]. Disponible: https://www.boe.es/buscar/ pdf/2002/BOE-A-2002-22188-consolidado.pdf

13. Gomollón F. Enfermedad inflamatoria intestinal. Gastroenterol Hepatol. 2013;31:42-6.

14. World Gastroenterology Organization. Guías Mundiales de la Organización Mundial de Gastroenterología. Enfermedad intestinal inflamatoria: actualización agosto de 2015. United States: World Gastroenterology Organization; 2015 [citado en 2021 Feb 14]. Disponible: https://www.worldgastroenterology.org/UserFiles/file/ guidelines/inflammatory-bowel-disease-spanish-2015.pdf
15. Enfermedad Inflamatoria Intestinal: situación actual y retos asistenciales. Londres: Ernst \& Young; 2016 [citado en 2018 Ago 1]. Disponible en: https://pt.slideshare.net/ernstandyoung/enfermedadinflamatoria-intestinal-situacin-actual-y-retos-asistenciales

16. Aldeguer $X$, Sicras-Mainar A. Costs of ulcerative colitis from a societal perspective in a regional health care area in Spain: A database study. Gastroenterol Hepatol. 2016;39(1):9-19.

17. Giorgi A, Giorgi B, Morley J. The Descriptive Phenomenological Psychological Method. In book: The Sage Handbook of Qualitative Research in Psychology. Edition: 2nd, Chapter: 11. London: Willig and Rogers; 2017. pp.176-92.

18. Kaplan CD, Korf D, Sterk C. Temporal and social contexts of heroinusing populations. An illustration of the snowball sampling technique. J Nerv Ment Dis. 1987;175(9):566-74.

19. López Cortes R. Discapacidad en la Enfermedad Inflamatoria Intestinal. Análisis de los efectos de la enfermedad en la vida cotidiana de los pacientes [tesis]. Granada: Universidad de Granada, Facultad de Ciencia s de la Salud; 2017 [citado en 2018 Ago 1]. Disponible en: http://digibug.ugr.es/bitstream/ handle/10481/45857/26504777.pdf?sequence=6\&isAllowed=y

20. Giorgi A. The theory, practice, and evaluation of the phenomenological method as a qualitative research procedure. J Phenomenol Psychol, 1997;28(2):235-60.

21. Ortega-Moreno M, Padilla-Garrido N, Huelva-López L, Aguado-Correa F, Bayo-Calero J, Bayo-Lozano E. Barreras y facilitadores para la implementación de la toma de decisiones compartidas en oncología: percepciones de los pacientes. Rev Calid Asist. 2017;32(3):141-5.

22. Veilleux S, Noiseux I, Lachapelle N, Kohen R, Vachon L, Guay BW, et al. Patients' perception of their involvement in shared treatment decision making: Key factors in the treatment of inflammatory bowel disease. Patient Educ Couns. 2018;101(2):331-9.

23. Légaré F, Adekpedjou R, Stacey D, Turcotte S, Kryworuchko J, Graham $I D$, et al. Interventions for increasing the use of shared decision making by healthcare professionals. Cochrane Database Syst Rev. 2018;7(7):CD006732. Review.

24. Joseph-Williams N, Elwyn G, Edwards A. Knowledge is not power for patients: a systematic review and thematic synthesis of patientreported barriers and facilitators to shared decision making. Patient Educ Couns. 2014;94(3):291-309. Review.

25. Wieringa TH, Rodriguez-Gutierrez R, Spencer-Bonilla G, de Wit M, Ponce 0J, Sanchez-Herrera MF, et al. Decision aids that facilitate elements of shared decision making in chronic illnesses: a systematic review. Syst Rev. 2019;8(1):121. Review. 